Article

\title{
Doxorubicin Release Controlled by Induced Phase Separation and Use of a Co-Solvent
}

\author{
Seok Chan Park ${ }^{1}$, Yue Yuan ${ }^{2}$ (1), Kyoungju Choi ${ }^{1}$, Seong-O Choi ${ }^{1,3}$ and Jooyoun Kim ${ }^{4,5, *}$ (1) \\ 1 Department of Anatomy and Physiology, Kansas State University, Manhattan, KS 66506, USA; \\ schpark@ksu.edu (S.C.P.); kjchoi@vet.k-state.edu (K.C.); sochoi@ksu.edu (S.-O.C.) \\ 2 Department of Textiles Engineering, Chemistry and Science, North Carolina State University, \\ Raleigh, NC 27695, USA; yyuan14@ncsu.edu \\ 3 Nanotechnology Innovation Center of Kansas State, Kansas State University, Manhattan, KS 66506, USA \\ 4 Department of Textiles, Merchandising and Fashion Design, Seoul National University, Seoul 08826, Korea \\ 5 Research Institute of Human Ecology, Seoul National University; Seoul 08826, Korea \\ * Correspondence: jkim256@snu.ac.kr; Tel.: +82-2-880-6846
}

Received: 25 March 2018; Accepted: 25 April 2018; Published: 26 April 2018

check for updates

\begin{abstract}
Electrospun-based drug delivery is emerging as a versatile means of localized therapy; however, controlling the release rates of active agents still remains as a key question. We propose a facile strategy to control the drug release behavior from electrospun fibers by a simple modification of polymer matrices. Polylactic acid (PLA) was used as a major component of the drug-carrier, and doxorubicin hydrochloride (Dox) was used as a model drug. The influences of a polar co-solvent, dimethyl sulfoxide (DMSO), and a hydrophilic polymer additive, polyvinylpyrrolidone (PVP), on the drug miscibility, loading efficiency and release behavior were investigated. The use of DMSO enabled the homogeneous internalization of the drug as well as higher drug loading efficiency within the electrospun fibers. The PVP additive induced phase separation in the PLA matrix and acted as a porogen. Preferable partitioning of Dox into the PVP domain resulted in increased drug loading efficiency in the PLA/PVP fiber. Fast dissolution of PVP domains created pores in the fibers, facilitating the release of internalized Dox. The novelty of this study lies in the detailed experimental investigation of the effect of additives in pre-spinning formulations, such as co-solvents and polymeric porogens, on the drug release behavior of nanofibers.
\end{abstract}

Keywords: poly(lactic acid); polyvinylpyrrolidone; dimethyl sulfoxide; doxorubicin; phase separation; co-solvent; porogen; drug release; electrospinning

\section{Introduction}

Over recent decades, electrospinning has been used as a versatile means of fabricating submicron fibers. A typical laboratory set-up of the electrospinning process consists of high voltage power supply, syringe, needle and conducting collector [1-3]. This old technique invented in the 1930s has regained academic and industrial attention with a growing interest in nanotechnology. As the fibrous web of nanofibers resembles the native extracellular matrix in its physical structure, electrospun nanofibers have been widely employed particularly in biomedical engineering. Especially, the high porosity of electrospun materials promotes the permeation of gases and nutrients [4-6] in tissue scaffolds, and high surface-to-volume ratio facilitates the adhesion and proliferation of cells [7,8]. With such characteristics, electrospun materials have become an attractive option for tissue engineering $[9,10]$ and prospective drug delivery systems $[8,11]$.

The main objective of drug delivery is to design a desired release profile while minimizing adverse side effects. To enhance the biocompatibility and reduce the major immune responses, a wide 
range of biocompatible polymers have been tested as carrier polymers [12-18]. For the fiber-based drug delivery, a drug is usually mixed with the carrier polymer in a pre-spinning solution. After the fibers are spun, drug molecules may exist as dispersions in a polymer matrix or be internalized like a monolithic polymer solution depending on the compatibility between the drug molecules and the carrier polymer. The release kinetics of a drug delivery system can be controlled by altering the solubility and swelling of carrier polymers. In the erosion-controlled system, the release can be promoted by the increased degradability and solubility of the carrier. In the diffusion-controlled system, the release can be promoted by the wettability and swelling of the carrier [19]. As hydrophobic materials such as poly(lactic-co-glycolic acid) (PLGA) [20-22] and polylactic acid (PLA) [18,23,24] poorly swell and slowly degrade in the human body, these materials tend to show a slow release of drugs without bioaccumulation [25-32]. On the contrary, fast biodegrading polymers such as gelatin [14-16] and polyvinylpyrrolidone [33] tend to exhibit immediate release of incorporated drugs.

To adjust the degradability and wettability of a polymeric matrix, polymer blends [34-36] or block copolymers [21,37] can be formulated by selective material combinations. For example, adding a water-soluble/swellable polymer additive in a hydrophobic carrier system can significantly increase the drug release rate [38]. In addition to modifying the system formulations, distinct release kinetics can be achieved by utilizing multilayered [35,39-41] or porous structures [41-43]. Environmental stimulations such as $\mathrm{pH}$, temperature, light, electrical field, and magnetic field [44-53] can also be used to manipulate the release at the specific target sites. While many of those methods often require sophisticated process modifications, in practical applications it is desirable to establish a simple strategy to manipulate the drug release.

The objective of this study is to develop a facile means of controlling drug release behavior by modifying the formulation of pre-spinning solutions. Polylactic acid (PLA) was used as a main polymeric drug-carrier, and doxorubicin $\mathrm{HCl}$ (Dox), a hydrophilic form of Dox, was used as a model drug. By adding $20 \mathrm{wt} \%$ of polyvinylpyrrolidone (PVP) in PLA matrix, the phase separation was purposely induced. Due to the hydrophilic nature of PVP, PVP acted as a porogen in the hydrophobic PLA matrix. The selective dissolution of PVP domains during the release stage created pores in the matrix, influencing the diffusivity of Dox in the medium. Furthermore, dimethyl sulfoxide (DMSO) was employed as a co-solvent of Dox, and its role on drug-polymer compatibility and release characteristics was investigated. The effect of DMSO on Dox-miscibility in a hydrophobic polymer was discussed using the concept of solubility parameters. The novelty of this study is in the detailed experimental investigation of drug release behavior in the presence of a hydrophilic co-solvent and a polymeric porogen. The findings of this research would provide a simple guide of controlling drug release behavior in a fiber-based drug delivery system, with a minimal modification of pre-spinning formulations.

\section{Materials and Methods}

\subsection{Materials}

Polylactic acid (PLA) resin (Ingeo 4043D, NatureWorks), 98\% L-lactide, with weight average molecular weight of $111 \mathrm{~kg} / \mathrm{mol}$ was purchased from NatureWorks (Minnetonka, MN, USA). Polyvinylpyrrolidone (PVP) with average molecular weight of $1300 \mathrm{~kg} / \mathrm{mol}$ was purchased from Alfa Aesar (Haverhill, MA, USA). Dimethyl sulfoxide (DMSO) and dimethylformamide (DMF) were purchased from Sigma-Aldrich (St. Louis, MO, USA). Doxorubicin hydrochloride (99\%, Dox- $\mathrm{HCl})$ was purchased from MedKoo Biosciences (Morrisville, NC, USA). Solvents, phosphate buffered saline (PBS, pH 7.4) were purchased from Thermo Fisher (Waltham, MA, USA), and other chemicals were purchased from Fisher Scientific (Hampton, NH, USA). 


\subsection{Preparation of Drug-Loaded Fiber Webs}

For PLA electrospinning, 9\% $(w / v)$, the PLA solution was prepared in a $1: 1$ ratio of dichloromethane (DCM) and dimethyl formamide (DMF). For PVP blending, 16\% (w/v) of PVP solution was prepared in DMF, and then this solution was mixed with the PLA solution in 80:20 weight ratio. For electrospinning (Spraybase ${ }^{\circledR}$, Dublin, Ireland), a grounded aluminum collector was placed in front of a 22-gauge needle at the distance of $10 \mathrm{~cm}$, and fibers were spun horizontally toward the collector rotating at $100 \mathrm{rpm}$. The feeding rate of polymer solution was $1.5 \mathrm{~mL} / \mathrm{h}$. The applied voltage was adjusted to $11 \mathrm{kV} 14 \mathrm{kV}$ to produce non-beaded fibers.

For drug loading, doxorubicin hydrochloride (Dox) was added in the polymer solutions (PLA and PLA/PVP blend) before electrospinning by two methods. One of the methods was to mix the drug particles directly to a PLA or a PLA/PVP pre-spinning solution, stirring at $100 \mathrm{rpm}$ for $1 \mathrm{~h}$ at room temperature. The formulation for PLA with Dox pre-spinning solution was: Dox 0.02 g, PLA 0.4 g, 2 mL DCM, 2 mL DMF. The formulation for PLA/PVP mixture was: Dox 0.02 g, PLA $0.315 \mathrm{~g}$ (10\% PLA solution $3.5 \mathrm{~mL}), 0.08 \mathrm{~g}$ PVP (16\% PVP solution $0.5 \mathrm{~mL})$. In the other method, Dox was firstly dissolved in DMSO by $2 \%(w / v)$, and $1 \mathrm{~mL}$ of Dox/DMSO solution was mixed with $4 \mathrm{~mL}$ of $9 \%(w / v)$ PLA solution (PLA-Dox(DMSO)) or $4 \mathrm{~mL}$ of PLA/PVP solution (PLA/PVP-Dox(DMSO)), respectively. The sample codes and descriptions are shown in Table 1.

Table 1. Sample descriptions.

\begin{tabular}{ll}
\hline \multicolumn{1}{c}{ Code } & \multicolumn{1}{c}{ Description } \\
\hline PLA & PLA web \\
PLA/PVP & PLA/PVP blend web (80:20 weight ratio) \\
PLA-Dox & Dox-loaded PLA web, Dox was mixed with PLA pre-spinning solution \\
PLA/PVP-Dox & Dox-loaded PLA/PVP web, Dox was mixed with PLA/PVP pre-spinning solution \\
PLA-Dox(DMSO) & Dox-loaded PLA web, Dox dissolved in DMSO was mixed with PLA pre-spinning solution \\
PLA/PVP-Dox(DMSO) & Dox-loaded PLA/PVP web, Dox dissolved in DMSO was mixed with PLA/PVP \\
& pre-spinning solution \\
\hline
\end{tabular}

\subsection{Characterization}

Optical and fluorescence images of Dox-loaded webs were observed with an inverted fluorescent microscope system (Olympus IX73, Olympus Corporation, Tokyo, Japan). Images were analyzed by ImageJ software (version 1.46r, NIH, Bethesda, MD, USA). Fiber morphology was observed by a scanning electron microscope (Merlin Compact FE-SEM, Zeiss, Oberkochen, Germany) with Pt sputter coating for $200 \mathrm{~s}$ (MSC-101, JEOL, Tokyo, Japan).

Static contact angles (CA) of $4 \mu \mathrm{L}$ of distilled water on fibrous surfaces were measured by an optical tensiometer (Attension Theta, Biolin Scientific, Paramus, NJ, USA) at room temperature.

\subsection{Solubility Parameter}

To explain the compatibility of polymers and solvents, Hansen solubility parameters from reference values were used (Table 2). The Hansen solubility parameter in Equation (1) is comprised of three parameters: energy from dispersion bonds between molecules, $\delta_{\mathrm{d}}$; dipolar intermolecular force between molecules, $\delta_{\mathrm{p}}$; and the hydrogen bonds between molecules, $\delta_{\mathrm{h}}$. The solubility parameter of mixture solvent was calculated using Equation (2). To estimate the compatibility between two components, the interaction parameter, R, was calculated by Equation (3). The calculated values of R's are presented in Table 3, where a smaller $\mathrm{R}$ represents a higher compatibility between components.

$$
\begin{gathered}
\delta_{\mathrm{t}}=\sqrt{\left(\delta_{\mathrm{d} 1}-\delta_{\mathrm{d} 2}\right)^{2}+\left(\delta_{\mathrm{p} 1}-\delta_{\mathrm{p} 2}\right)^{2}+\left(\delta_{\mathrm{h} 1}-\delta_{\mathrm{h} 2}\right)^{2}}, \\
\delta_{\text {mix }}=\mathrm{f}_{1} \cdot \delta_{1}+\mathrm{f}_{2} \cdot \delta_{2}, \\
\mathrm{R}=\sqrt{4\left(\delta_{\mathrm{d} 1}-\delta_{\mathrm{d} 2}\right)^{2}+\left(\delta_{\mathrm{p} 1}-\delta_{\mathrm{p} 2}\right)^{2}+\left(\delta_{\mathrm{h} 1}-\delta_{\mathrm{h} 2}\right)^{2}},
\end{gathered}
$$


$\delta_{\mathrm{t}}\left(\mathrm{MPa}^{1 / 2}\right)$ : total solubility parameter; $\delta_{\mathrm{d}}\left(\mathrm{MPa}^{1 / 2}\right)$ : dispersion force component; $\delta_{\mathrm{p}}\left(\mathrm{MPa}^{1 / 2}\right)$ : polar force component; $\delta_{\mathrm{h}}\left(\mathrm{MPa}^{1 / 2}\right)$ : hydrogen-bonding component; $\delta_{1}$ and $\delta_{2}$ : solubility of the components 1 and 2 , respectively; $\delta_{\text {mix }}\left(\mathrm{MPa}^{1 / 2}\right)$ : solubility parameter of the mixture of components 1 and $2 ; f_{1}$ and $f_{2}$ : volume ratio of the components 1 and $2 ; \mathrm{R}\left(\mathrm{MPa}^{1 / 2}\right)$ : interaction parameter.

Table 2. Hansen solubility parameters.

\begin{tabular}{ccccc}
\hline Component & $\boldsymbol{\delta}_{\mathbf{t}} \mathbf{( \mathbf { M P a } ^ { \mathbf { 1 / 2 } } )}$ & $\boldsymbol{\delta}_{\mathbf{d}} \mathbf{( \mathbf { M P a } ^ { \mathbf { 1 / 2 } } )}$ & $\boldsymbol{\delta}_{\mathbf{p}} \mathbf{( \mathbf { M P a } ^ { \mathbf { 1 / 2 } } )}$ & $\boldsymbol{\delta}_{\mathbf{h}} \mathbf{( \mathbf { M P a } ^ { \mathbf { 1 / 2 } } )}$ \\
\hline PLA [54] & 21.2 & 17.5 & 9.5 & 7.3 \\
PVP [55] & 24.3 & 18.8 & 13.4 & 7.5 \\
DMSO [56] & 26.7 & 18.4 & 16.4 & 10.2 \\
DCM [56] & 20.2 & 18.2 & 6.3 & 6.1 \\
DMF [56] & 24.9 & 17.4 & 13.7 & 11.3 \\
DCM/DMF * & 22.2 & 17.8 & 10.0 & 8.7 \\
\hline \multicolumn{5}{c}{ * Calculated value for 1:1 ratio of DCM:DMF. }
\end{tabular}

Table 3. Interaction parameters $\mathrm{R}$ between polymer and solvents.

\begin{tabular}{ccccc}
\hline $\mathbf{R}\left(\mathbf{M P a}^{\mathbf{1} \mathbf{2}}\right)$ & DMSO & DCM & DMF & DCM/DMF \\
\hline PLA & 7.7 & 3.7 & 5.8 & 1.6 \\
PVP & 4.1 & 7.3 & 4.7 & 4.1 \\
DMSO & 0 & 10.9 & 3.5 & 6.7 \\
\hline
\end{tabular}

\subsection{Phase Separation}

Phase separation of PLA and PVP blends was observed to investigate the drug distribution between PLA and PVP regions. The $5 \mu \mathrm{L}$ pre-spinning solutions with and without drug were loaded between two glass coverslips, and observed under the inverted fluorescent microscope system. The solution compositions for the phase separation study were maintained the same as the pre-spinning solutions to simulate the phase separation occurring during web formation.

\subsection{Loading Content (LC) and Loading Efficiency (LE)}

Drug loading content (LC\%) was determined by the weight percentage of a drug in a fiber web (Equation (4)). For this measurement, drug-loaded fiber webs were cut into rectangular strips and weighed. The samples were completely dissolved in $2.5 \mathrm{~mL}$ of chloroform and mixed vigorously with $5 \mathrm{~mL}$ of deionized water (DI water). The mixtures were stored in dark overnight for spontaneous phase separation of organic and aqueous phases. The supernatant was taken after centrifugation at 4696× $\mathrm{g}$ and diluted 10-fold for Ultraviolet/Visible (UV/Vis) spectroscopy (Synergy H1 Hybrid Multi-Mode Reader, BioTek, Winooski, VT, USA). The number of drugs in the web were calculated from the triplicate measurements of absorbance of Dox at $480 \mathrm{~nm}$. Loading efficiency (LE\%) of a drug-loaded fiber web was calculated by Equation (5).

$$
\begin{gathered}
\mathrm{LC}(\%)=\frac{\text { weight of drug loaded in the web }(\mathrm{mg})}{\text { weight of the drug loaded fiber web }(\mathrm{mg})} \times 100(\%), \\
\mathrm{LE}(\%)=\frac{\text { weight of drug loaded in the web }(\mathrm{mg})}{\text { weight of drug initally added in the polymer solution }(\mathrm{mg})} \times 100(\%) .
\end{gathered}
$$

\subsection{In-Vitro Drug Release Analysis}

About $20 \mathrm{mg}$ of a drug-loaded web was immersed in $3.5 \mathrm{~mL}$ of phosphate buffered saline (PBS, $\mathrm{pH}$ 7.4) at $37^{\circ} \mathrm{C}$ and was stirred at $100 \mathrm{rpm}$ using a vertical diffusion cell (Perme Gear, Hellertown, PA, USA). At each sampling point, the full amount of $3.5 \mathrm{~mL}$ of drug-released PBS medium was replaced with fresh $3.5 \mathrm{~mL}$ PBS. To measure the amount of drug released from the fiber composites, 
the fluorescence intensity of doxorubicin $(590 \mathrm{~nm})$ was measured using a UV-visible microplate reader (Synergy H1 Hybrid Multi-Mode Reader, BioTek) with an excited wavelength of $480 \mathrm{~nm}$. The amount of drug released from the web at each sampling interval was quantified by the calibration curve for fluorescence intensities of Dox standard concentrations. The cumulative percentage of released drug at each sampling point was calculated by Equation (6). All experiments were conducted with at least three replications.

$$
\text { cumulative drug release }(\%)=\frac{\sum_{t_{0}}^{t_{n}} \text { weight of drug released }(\mathrm{mg})}{\text { weight of drug in the web }(\mathrm{mg})} \times 100(\%),
$$

$t_{0}$ : The initial collection point at release time $=0 \mathrm{~h} ; \mathrm{t}_{\mathrm{n}}$ : The $\mathrm{nth}$ collection point at release time $=\mathrm{n} \mathrm{h}$.

\section{Results}

\subsection{Drug Localization in the Drug-Carrier}

The influence of immiscible polymer blending on drug localization in the fiber-based drug-carrier was observed by optical and fluorescence microscopies (Figure 1). Doxorubicin $\mathrm{HCl}$ used in this study has hydrophilic nature and can be dissolved in hydrophilic solvents such as water and DMSO. When Dox was directly added to a hydrophobic PLA pre-spinning solution (PLA in DCM/DMF), Dox was not soluble; instead, its particles were aggregated and precipitated in the solution. Due to the lack of compatibility between Dox and PLA, Dox-incorporating fibers produced large Dox aggregates as observed from the optical and fluorescence images in Figure 1b,c. Comparing fluorescence images of PLA-Dox and PLA/PVP-Dox (Figure 1c), PLA-Dox fibers were rarely fluorescent, while PLA/PVP-Dox fibers were fluorescent resulting from the incorporated Dox. For PLA/PVP-Dox samples, Dox appears to be mostly internalized in the hydrophilic PVP domains rather than PLA domains. From the fact that Dox particles still remained in the PLA/PVP-Dox web, 20\% of PVP content may not be sufficient to fully internalize the added Dox (loading content $\sim 3.1 \%$ ).
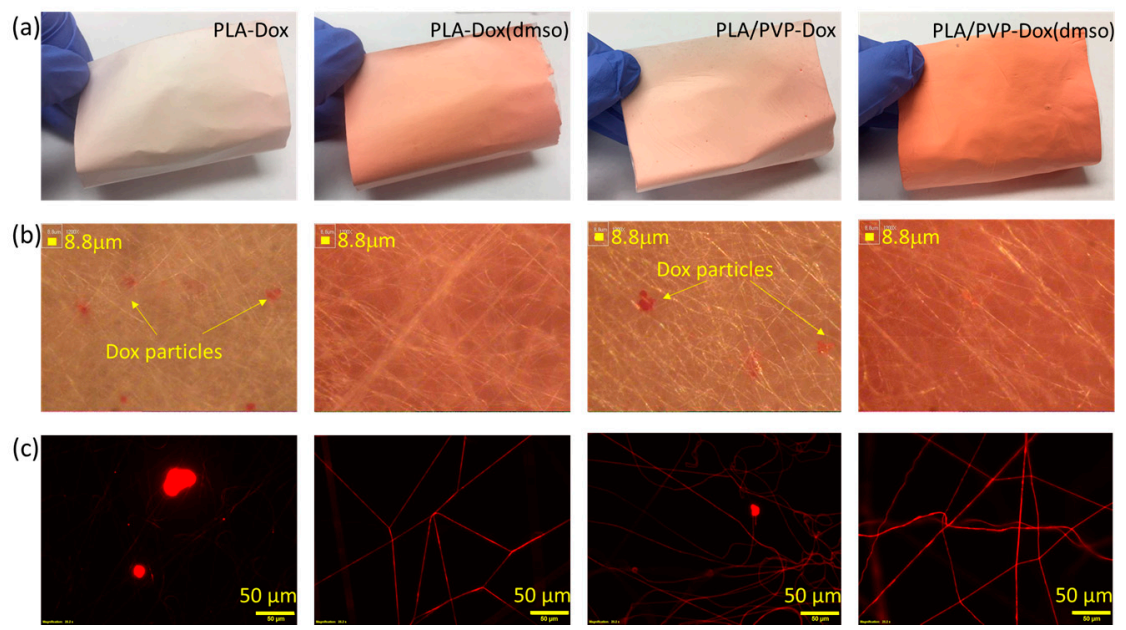

Figure 1. Doxorubicin-loaded fiber webs. (a) Photographic images of drug-loaded webs; (b) optical images displaying drug aggregates; (c) fluorescence images with Dox-loaded webs.

When Dox was dissolved in DMSO, Dox was homogeneously internalized in the fibers, leaving little Dox particles, and the fibers produced more saturated color. From the microscopic images, when Dox was dissolved in DMSO, the miscibility of Dox in hydrophobic PLA appeared to be significantly improved, with the enhanced interaction between Dox/DMSO and PLA solution. Likewise, PLA/PVP-Dox(DMSO) exhibited good compatibility between Dox and PLA with little Dox particles. From the solubility parameters of solvents, the interaction parameter R between PVP and DMSO was calculated to be about 4.1 (Table 3), which is smaller than that of PLA and DMSO; 
this implies that DMSO is more compatible with PVP than with PLA. Thus, in a PLA/PVP blend, DMSO would favor partitioning in PVP over PLA. The compatibility among Dox, PLA and PVP was further examined by casting polymeric films.

\subsection{Phase Separation Between PLA and PVP}

The phase separation was observed from the polymer blends loaded on glass coverslips (Figure 2) [34,35]. For PLA/PVP blends, 20\% of PVP created its domains in the blend matrices by phase separation, due to the lack of compatibility between PLA and PVP (Figure 2b). When Dox was added to a PLA/PVP blend without DMSO, Dox was either dissolved in PVP domains or formed aggregates in the bulk (Figures $2 \mathrm{~d}$ and 3b, PLA/PVP-Dox). When Dox was dissolved in DMSO, Dox was well-blended in both PVP and PLA domains, while a clear phase separation was still observed (Figures $2 \mathrm{~g}$ and $3 \mathrm{e}$ ). When DMSO was added, the size of PVP domains was considerably reduced, producing smaller emulsions due to the enhanced stability of the mixture.

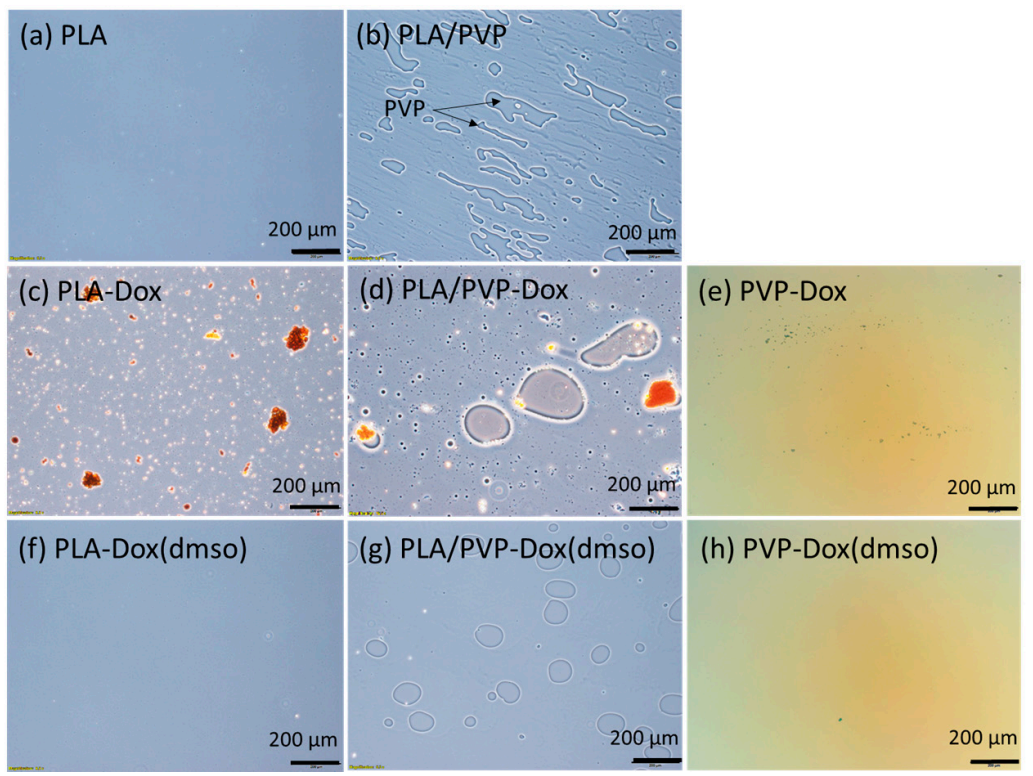

Figure 2. Microscopy images of polylactic acid (PLA) solution with homogenous single phase and PLA/polyvinylpyrrolidone (PVP) solution with phase separation.

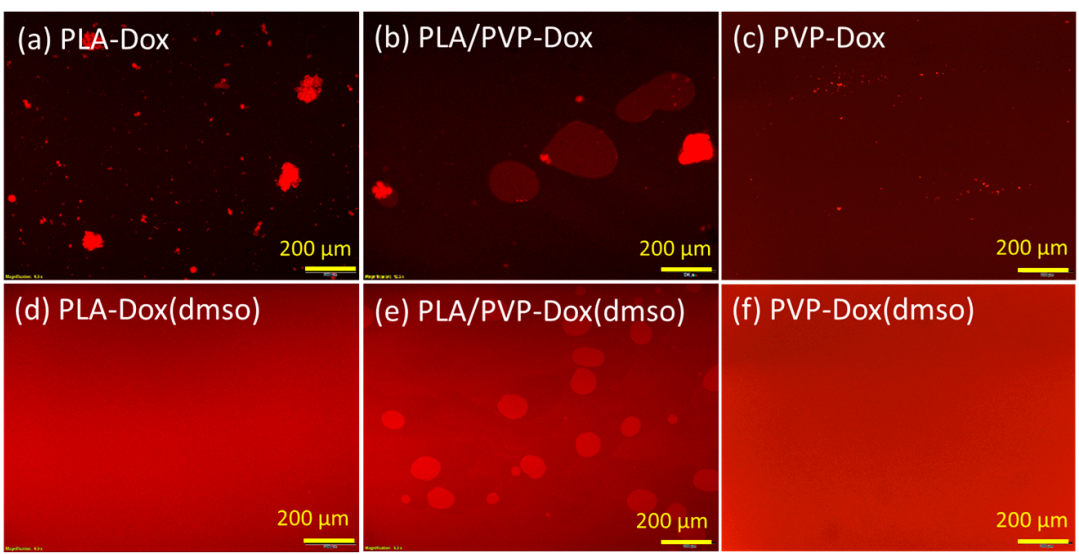

Figure 3. Fluorescence microscopy images of PLA-doxorubicin hydrochloride (Dox), PLA-Dox(dimethyl sulfoxide (DMSO)), PLA/PVP-Dox and PLA/PVP-Dox(DMSO) pre-spinning solutions casted on glass slides.

For a PLA-only matrix, Dox particles formed large aggregates (Figures 2c and 3a, PLA-Dox); with the aid of DMSO, Dox was homogeneously dissolved like a monolithic matrix (Figures $2 \mathrm{f}$ and $3 \mathrm{~d}$, 
PLA-Dox(DMSO)). Little or no fluorescence was observed from PLA background (Figure 1c), implying Dox was not well internalized in PLA matrix without an aid of DMSO. The hydrophilic Dox itself (without DMSO) was blended better into PVP (Figures 2e and 3c) than into PLA, yet small Dox particles remained in the PVP matrix. DMSO enhanced the miscibility of Dox in the PVP-only matrix also, leaving little particles in the bulk matrix (Figures $2 \mathrm{~h}$ and $3 \mathrm{f}$ ).

\subsection{Morphological Influence of Fibers Before and After Immersion in Water}

The morphology of fiber webs is shown in Figure 4. When DMSO was added to PLA and PLA/PVP, small pores were formed on the fiber surfaces; the size of pores ranged from tens of nanometers to about $300 \mathrm{~nm}$ when measured by ImageJ. As the volatility of DMSO (vapor pressure $\sim 59 \mathrm{~Pa}$ at $20^{\circ} \mathrm{C}$ ) is considerably lower than that of DCM (vapor pressure $\sim 47,400 \mathrm{~Pa}$ at $20^{\circ} \mathrm{C}$ ) and $\mathrm{DMF}$ (vapor pressure $\sim 380 \mathrm{~Pa}$ at $20^{\circ} \mathrm{C}$ ), DMSO remains on a fiber surface longer than other solvents when fibers solidify upon solvent evaporation. When DMSO finally evaporates from the fiber surface, pores can be created on the surface, as observed in Figure 4c.

To investigate the morphological changes of fibers with water immersion, fibrous webs were immersed in distilled water for $24 \mathrm{~h}$ at $37^{\circ} \mathrm{C}$ and observed by SEM (Figure 5). For PLA/PVP fibers, the hydrophilic PVP acted as a porogen in hydrophobic PLA matrix, and after $24 \mathrm{~h}$ immersion, pores were created (in tens of nanometers to about $300 \mathrm{~nm}$ ) on the fiber surface; on the other hand, PLA fibers did not show noticeable morphological changes. The influence of these pore formation on the Dox-release profile is discussed in a later section.

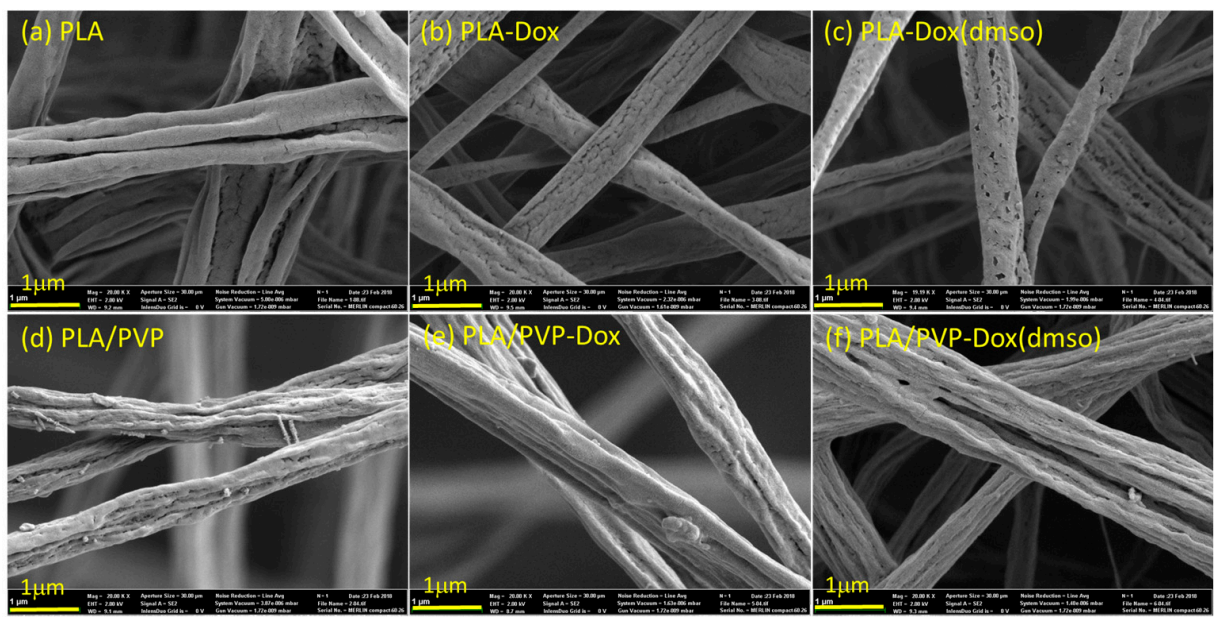

Figure 4. SEM images of doxorubicin-loaded fibers.

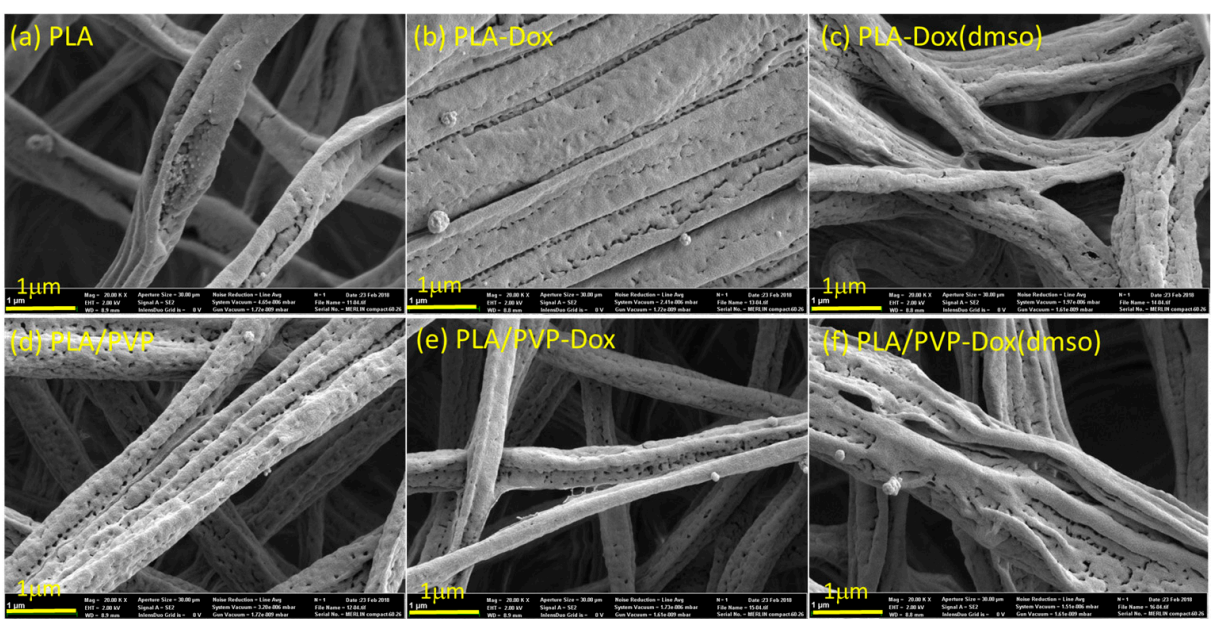

Figure 5. SEM images of doxorubicin-loaded fibers after immersion in water for $24 \mathrm{~h}$. 


\subsection{Wettability of Drug-Loaded Webs}

The influence of PVP blending to PLA on wettability was examined by water contact angle (CA) measurement (Figure 6). The CA of pristine PLA web without drug loading was $160^{\circ}$. When Dox was added to PLA (PLA-Dox), CA decreased to about $139^{\circ}$. The wetting property was not uniform because the CA measurement varied depending on the presence of Dox aggregates on the measured locations. When Dox was uniformly blended in PLA matrix with the aid of co-solvent DMSO, surface wettability turned more uniform with CA about $148^{\circ}$. With the addition of PVP (PLA/PVP and PLA/PVP-Dox), CA dropped to $114^{\circ} \sim 131^{\circ}$; also, the CA meausrements had a large variation depending on the size of PVP domains on the surface. When DMSO was added to the PLA/PVP blend (PLA/PVP-Dox(DMSO)), surface homogeneity was improved and the measured CA was consistent $\left(\sim 149^{\circ}\right)$.

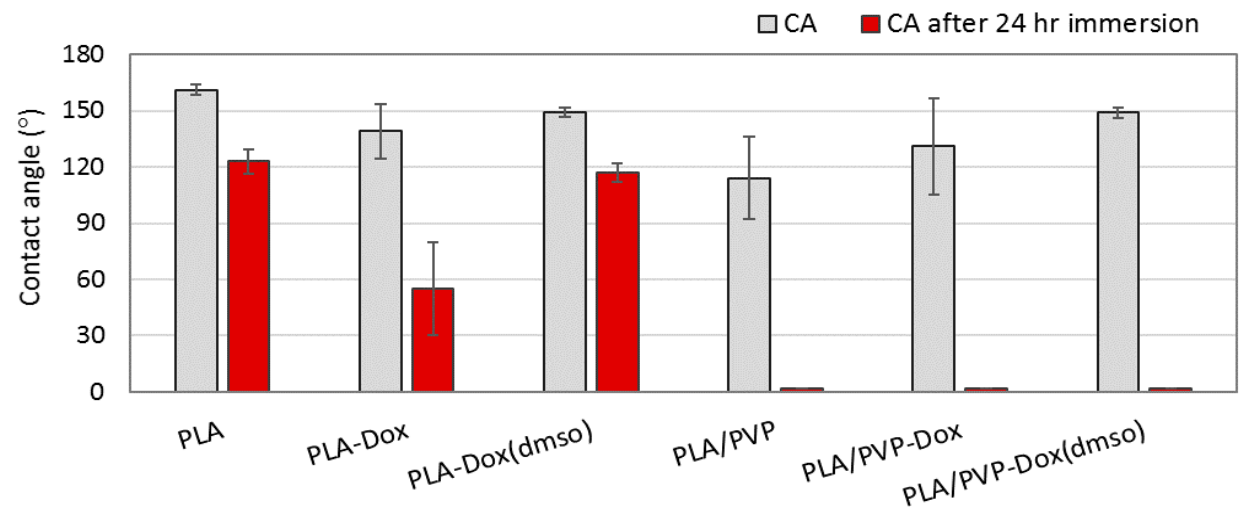

Figure 6. Contact angle of Dox-loaded fiber webs before and after immersion in water for $24 \mathrm{~h}$.

To examine how the wettability changes with drug release, CA was measured after $24 \mathrm{~h}$ of water immersion at $37^{\circ} \mathrm{C}$. The immersed webs were completely dried before $\mathrm{CA}$ re-measurement. After the immersion, PLA without Dox loading $\left(\mathrm{CA} \sim 123^{\circ}\right.$ ) and monolithic-like PLA-Dox(DMSO) (CA $\sim 117^{\circ}$ ) maintained its hydrophobicity. However, wettability of PLA-Dox was considerably increased after the immersion. For all PVP blended samples, wettability was significantly enhanced after the immersion, where the water drop was immediately spread on the surface $\left(\mathrm{CA} \sim 0^{\circ}\right)$. From Figure $5 \mathrm{~d}-\mathrm{f}, \mathrm{PVP}$ domains of the fibers began to dissolve upon water exposure, creating pores. The dissolved PVP may have coated the fiber surface, turning the surface more hydrophilic. Also, the irregular surface roughness resulting from pores and wrinkles, combined with the increased hydrophilicity, contributed to further increase of wettability according to the Wenzel theory [57].

\subsection{Loading Contents (LC) and Loading Efficiency (LE)}

The loading content (LC) and loading efficiency (LE) of Dox were examined (Figure 7). PLA-Dox, in which Dox was not homogeneously blended with PLA, showed only 59\% LE, producing the actual drug content of $2.9 \%(w / w)$. When hydrophilic Dox was blended with PLA solution, the system was not stable; Dox formed aggregates and precipitated in the solution. The precipitates of Dox particles were visible at the bottom of the pre-spinning solution, and those Dox precipitates were not fully incorporated in the fibers during the spinning process. Thus, the incompatibility between the hydrophilic drug and the hydrophobic carrier polymer led to low drug loading efficiency and content.

With DMSO, Dox was well mixed with the PLA matrix, and there were no precipitates formed. For PLA-Dox(DMSO), LE of Dox increased up to about 91\%, forming a homogeneous, monolithic-like matrix with LC about $4.6 \%$. When a hydrophilic PVP polymer additive was mixed with PLA matrix, Dox appeared to dissolve mostly in PVP domains (Figure 3b), demonstrating the favorable partitioning of a hydrophilic drug into a hydrophilic polymer. Compared to PLA-Dox, PLA/PVP-Dox showed the enhanced Dox loading with LE of about 69\% (LC 3.5\%). When DMSO was used as a co-solvent of 
Dox in the PLA/PVP, the LE was about $86 \%$. As the solubility parameter of DMSO is closer to PVP than PLA, it was expected that loading of Dox/DMSO would be more efficient in PLA/PVP than in PLA. However, the results did not show this trend; on the contrary, LE\% of Dox/DMSO in PLA/PVP blend was slightly lower than PLA-Dox(DMSO). While the information is missing to explain this phenomenon, it can be concluded that the overall loading efficiency was enhanced by the addition of hydrophilic polymer additive and the hydrophilic co-solvent.
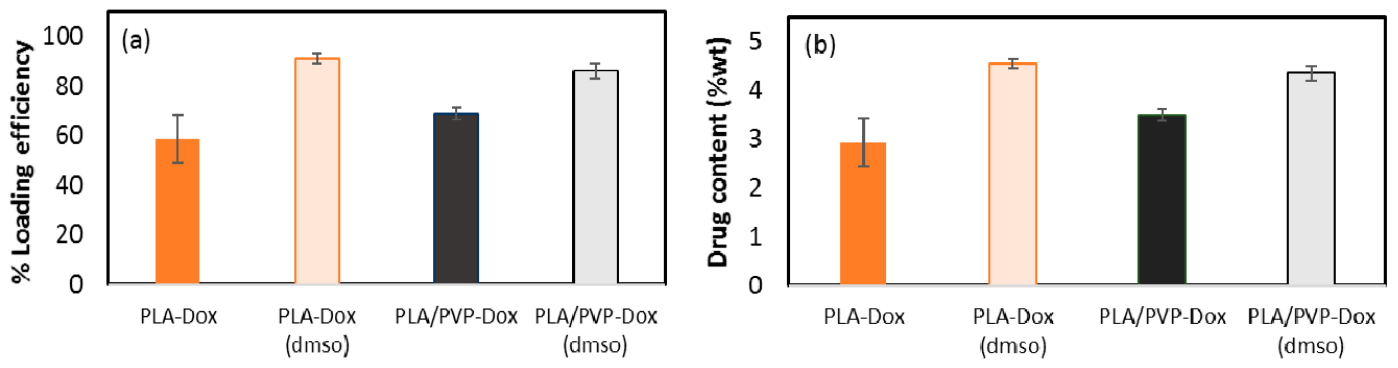

Figure 7. Loading content (a) and loading efficiency (b) of doxorubicin in the web.

\subsection{In-Vitro Dox Release}

From the in-vitro drug release in Figure 8, Dox dissolved in PLA with DMSO exhibited the distinct sustained release. The use of DMSO as a co-solvent considerably improved the miscibility of Dox and PLA, producing a homogeneous monolithic-like matrix. As PLA is rarely swelling in water, the internalized Dox in the monolithic-like fiber can hardly be diffused out from the polymer matrix; thus, the release of internalized Dox would be dependent mostly on PLA degradation. As is shown in SEM images of Figure 5, PLA fiber morphology had little changes after $24 \mathrm{~h}$ water immersion. As PLA degradation takes months of period, the release of Dox from the monolithic-like fibers would take as long as several months.

Rapid release with initial burst profile was shown by PLA-Dox, PLA/PVP-Dox, and PLA/PVP-Dox(DMSO) fibers. PLA-Dox, in which Dox formed aggregates, exhibited faster release in the first $5 \mathrm{~h}$ (about $60 \%$ ). The release during this period is attributed to the direct dissolution of Dox particles that are exposed to web surface. The Dox particles mostly existed as aggregates on the fiber surfaces due to the lack of compatibility between Dox and PLA. The release profile of PLA-Dox exhibited a large variation as a result of inhomogeneous dispersions of Dox crystals over the fiber surfaces. Dox in PLA was released up to about $80 \%$ of the total content till 3 days, which indicates that about $80 \%$ of Dox is distributed near the surface of PLA fibers. The remaining $20 \%$ Dox may exist inside PLA matrix, and the release of this portion would occur with PLA degradation.

PLA/PVP-Dox and PLA/PVP-Dox(DMSO) displayed similar release profiles. As Dox was preferentially dissolved in PVP over PLA domains, the release in the early stage is mostly attributed to PVP dissolution in PBS solution. The preferential distribution of Dox/DMSO in PVP over PLA domains can be validated by the lower interaction parameter, R between DMSO and PVP $\left(4.1 \mathrm{MPa}^{1 / 2}\right)$ than $\mathrm{R}$ between DMSO and PLA (7.7 $\mathrm{MPa}^{1 / 2}$ ) (Table 3). The higher release rate of PLA/PVP than that of PLA suggests that the selective dissolution of water-soluble PVP is a predominant factor of the release. Particularly, the burst release within $1 \mathrm{~h}$ is attributed to the fast dissolution of PVP domains with the incorporated Dox (Figure 8, enlarged plots); this selective dissolution is well observed from the pores created on the fiber surface after $24 \mathrm{~h}$ immersion (SEM images in Figure 5). For PLA/PVP-Dox(DMSO), $55 \%$ of cumulative release during the initial $3 \mathrm{~h}$ is attributed to the dissolution of PVP domains containing Dox. About 40-45\% of the total drug content distributed in PLA domains would be released along with PLA degradation.

In practical drug delivery applications, the amount of drug released, rather than the percentage of the loaded, may be more important. Considering the loading efficiency and the release rates $(\%)$, the Dox amount $(\mu \mathrm{g})$ released from $1 \mathrm{mg}$ of web was calculated (Figure $8 \mathrm{~b})$. From the 
results, PLA/PVP-Dox(DMSO) showed the highest amount of drug release ( $\mu \mathrm{g}$ Dox/mg web) during the first $24 \mathrm{~h}$ of release. While the released percentage of the total loaded was similar between PLA/PVP-Dox and PLA/PVP-Dox(DMSO), the total released amount ( $\mu \mathrm{g}$ Dox/mg web) was considerably higher for PLA/PVP-Dox(DMSO) ( $\sim .5 \mathrm{mg})$ than PLA/PVP-Dox ( 0.38 mg), due to the enhanced loading efficiency with DMSO. With low LE\% of PLA-Dox (2.9\%), the amount of Dox released from PLA-Dox was lower than that of PLA/PVP-Dox(DMSO). The amount of Dox released from PLA-Dox(DMSO) was still the lowest while the LE\% was as high as $91 \%$. Regarding in-vivo perspectives, PLA/PVP-Dox(DMSO) may also be advantageous for reaching the necessary therapeutic levels faster than others tested by releasing the highest dosage in the first $5 \mathrm{~h}$. As Dox incorporated in PLA would be released with PLA degradation, if enzymatic degradation of PLA is triggered in the body, Dox would be released faster under in-vivo conditions compared to in-vitro release. For effective treatments, it is necessary to load a sufficient amount of drugs and release at a controlled rate. Our data suggest that the addition of DMSO in the polymeric system increased the Dox loading by enhancing miscibility/compatibility in the polymeric matrix and could alter drug release rates.
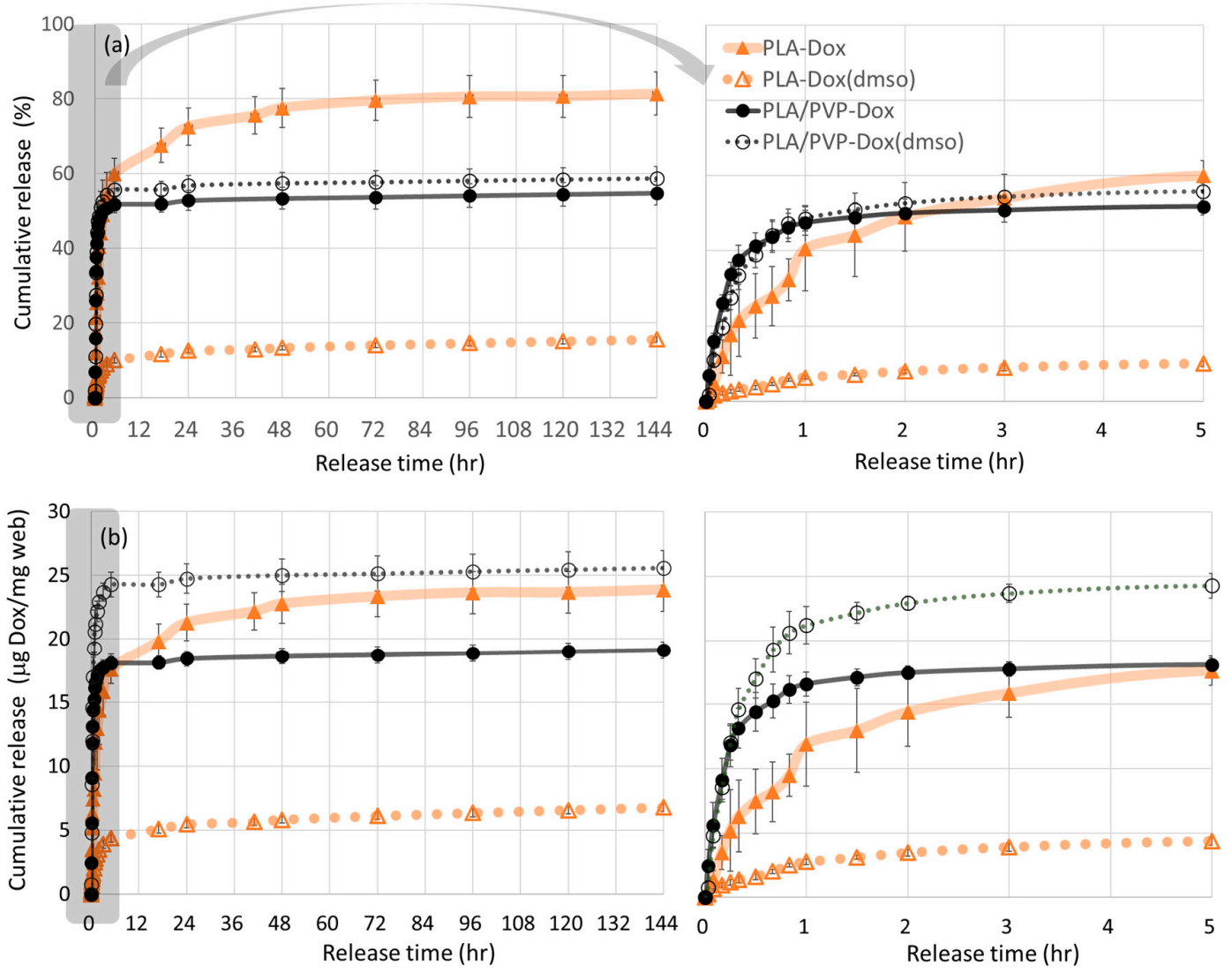

Figure 8. In-vitro 6-day release profile of doxorubicin from PLA and PLA/PVP webs. (a) Cumulative $\%$ release of Dox (left, release during $144 \mathrm{~h}$; right, enlarged for $5 \mathrm{~h}$ ); (b) cumulative released amount (mg) of Dox (left, release during $144 \mathrm{~h}$; right, enlarged for $5 \mathrm{~h}$ ).

While the compatibility of Dox both in PVP and PLA was enhanced with the use of DMSO, the effect on the release rate was completely different. For PLA-Dox(DMSO), the addition of DMSO lowered the release rates despite the improved Dox miscibility in PLA matrix, indicating that increased drug miscibility in a hydrophobic, slowly-degrading polymer matrix would retard the release of drugs. On the contrary, the use of DMSO in PLA/PVP blend further promoted the loading efficiency and release. The increased drug miscibility and solubility in a hydrophilic, fast-degrading hydrophilic polymer accelerated the drug release. The results demonstrate that the release of doxorubicin can be conveniently manipulated by the selection of polymer additives and solvents system. 


\section{Conclusions}

The release of a hydrophilic drug, doxorubicin hydrochloride (Dox), incorporated in electrospun PLA fibers was investigated along with drug loading efficiency to develop a facile strategy for controlling drug release from fiber-based drug delivery systems. Influences of miscibility and compatibility between the drug and polymer matrix on the loading and release properties were studied by adding a co-solvent and/or a hydrophilic polymer additive. A polar organic solvent, DMSO, and water-soluble polymer, PVP, were employed to enhance the miscibility of drug in a hydrophobic carrier polymer. In general, drug loading efficiency (LE) was improved by the addition of DMSO and PVP. The use of DMSO enabled the homogeneous incorporation of Dox, as well as higher drug loading, within the electrospun fibers.

Distinct drug release behaviors were demonstrated by use of DMSO and PVP. In the monolithic PLA fibers, the addition of DMSO helped homogeneous encapsulation of Dox in PLA matrix, and this led to the delayed Dox release. Without DMSO, Dox formed inhomogeneous dispersions on the PLA fiber surfaces, resulting in the burst release. When PVP was added in the polymer system, preferable partitioning of Dox into the PVP domains occurred, and this resulted in the enhanced loading of Dox in PLA/PVP fibers. The hydrophilic PVP additive acted as a porogen in the PLA/PVP system, and the porous morphology formed by the selective dissolution of PVP promoted the diffusion of the drug in a short time period.

In this study, doxorubicin release from nanofibers was manipulated by the addition of a hydrophilic porogen, the induced phase separation, and the use of co-solvent. The results suggest the importance of individual roles of solvent systems and polymer additives on drug release profiles. The addition of a compatible solvent to a polymer matrix enhanced drug loading efficiency and incorporation. However, the effect of drug-carrier miscibility on release rate was different for polymer types. The enhanced drug miscibility in a hydrophobic PLA matrix delayed the release, while the enhanced drug miscibility in a hydrophilic PVP matrix accelerated the release. The experimental investigation of this study confirmed the significance of the pre-spinning formulation in controlling the drug release rates. An extended study is recommended to test biocompatibility, in-vitro and in-vivo cancer cell viability to validate the effectiveness of the developed system.

Author Contributions: Jooyoun Kim and Seong-O Choi conceived and designed the experiments. Seok Chan Park, Yue Yuan, Kyoungju Choi, Seong-O Choi and Jooyoun Kim performed experiments. Jooyoun Kim, Seok Chan Park, Seong-O Choi, and Yue Yuan analyzed the data and wrote the paper.

Funding: This research was funded by Seoul National University (Research Resettlement Fund for the new faculty of Seoul National University) grant number [350-20170057].

Acknowledgments: Authors acknowledge the Research Institute of Advanced Materials of the Seoul National University for SEM images.

Conflicts of Interest: The authors declare no conflict of interest. The founding sponsors had no role in the design of the study; in the collection, analyses, or interpretation of data; in the writing of the manuscript, and in the decision to publish the results.

\section{References}

1. Di, J.; Zhao, Y.; Yu, J. Fabrication of molecular sieve fibers by electrospinning. J. Mater. Chem. 2011, 21, 8511-8520. [CrossRef]

2. Kim, J.S.; Reneker, D.H. Polybenzimidazole nanofiber produced by electrospinning. Polym. Eng. Sci. 1999, 39, 849-854. [CrossRef]

3. Li, D.; Xia, Y.N. Direct fabrication of composite and ceramic hollow nanofibers by electrospinning. Nano Lett. 2004, 4, 933-938. [CrossRef]

4. Ji, S.; Li, Y.; Yang, M. Gas sensing properties of a composite composed of electrospun poly(methyl methacrylate) nanofibers and in situ polymerized polyaniline. Sens. Actuators B: Chem. 2008, 133, 644-649. [CrossRef] 
5. Truong, Y.B.; O’Bryan, Y.; McKelvie, I.D.; Kyratzis, I.L.; Humphries, W. Application of Electrospun Gas Diffusion Nanofibre-membranes in the Determination of Dissolved Carbon Dioxide. Macromol. Mater. Eng. 2013, 298, 590-596. [CrossRef]

6. Saha, K.; Butola, B.S.; Joshi, M. Drug-Loaded Polyurethane/Clay Nanocomposite Nanofibers for Topical Drug- Delivery Application. J. Appl. Polym. Sci. 2014, 131, 40230. [CrossRef]

7. Huang, Z.; Zhang, Y.; Kotaki, M.; Ramakrishna, S. A review on polymer nanofibers by electrospinning and their applications in nanocomposites. Composit. Sci. Technol. 2003, 63, 2223-2253. [CrossRef]

8. Bhardwaj, N.; Kundu, S.C. Electrospinning: A fascinating fiber fabrication technique. Biotechnol. Adv. 2010, 28, 325-347. [CrossRef] [PubMed]

9. Matthews, J.A.; Wnek, G.E.; Simpson, D.G.; Bowlin, G.L. Electrospinning of collagen nanofibers. Biomacromolecules 2002, 3, 232-238. [CrossRef] [PubMed]

10. Li, W.J.; Laurencin, C.T.; Caterson, E.J.; Tuan, R.S.; Ko, F.K. Electrospun nanofibrous structure: A novel scaffold for tissue engineering. J. Biomed. Mater. Res. 2002, 60, 613-621. [CrossRef] [PubMed]

11. Sill, T.J.; von Recum, H.A. Electro spinning: Applications in drug delivery and tissue engineering. Biomaterials 2008, 29, 1989-2006. [CrossRef] [PubMed]

12. Becker, T.; Kipke, D.; Brandon, T. Calcium alginate gel: A biocompatible and mechanically stable polymer for endovascular embolization. J. Biomed. Mater. Res. 2001, 54, 76-86. [CrossRef]

13. Orive, G.; Ponce, S.; Hernandez, R.; Gascon, A.; Igartua, M.; Pedraz, J. Biocompatibility of microcapsules for cell immobilization elaborated with different type of alginates. Biomaterials 2002, 23, 3825-3831. [CrossRef]

14. Dhandayuthapani, B.; Krishnan, U.M.; Sethuraman, S. Fabrication and characterization of chitosan-gelatin blend nanofibers for skin tissue engineering. J. Biomed. Mater. Res. Part B: Appl. Biomater. 2010, 94, 264-272. [CrossRef] [PubMed]

15. Huang, C.; Chi, C.; Chen, Y.; Chen, K.; Chen, P.; Yao, C. Evaluation of proanthocyanidin-crosslinked electrospun gelatin nanofibers for drug delivering system. Mater. Sci. Eng. C: Mater. Biol. Appl. 2012, 32, 2476-2483. [CrossRef]

16. Huang, Z.; Zhang, Y.; Ramakrishna, S.; Lim, C. Electrospinning and mechanical characterization of gelatin nanofibers. Polymer 2004, 45, 5361-5368. [CrossRef]

17. Mo, X.; Xu, C.; Kotaki, M.; Ramakrishna, S. Electrospun P(LLA-CL) nanofiber: A biomimetic extracellular matrix for smooth muscle cell and endothelial cell proliferation. Biomaterials 2004, 25, 1883-1890. [CrossRef] [PubMed]

18. Yang, F.; Murugan, R.; Ramakrishna, S.; Wang, X.; Ma, Y.; Wang, S. Fabrication of nano-structured porous PLLA scaffold intended for nerve tissue engineering. Biomaterials 2004, 25, 1891-1900. [CrossRef] [PubMed]

19. Sebe, I.; Szabo, P.; Kallai-Szabo, B.; Zelko, R. Incorporating small molecules or biologics into nanofibers for optimized drug release: A review. Int. J. Pharm. 2015, 494, 516-530. [CrossRef] [PubMed]

20. Bashur, C.A.; Dahlgren, L.A.; Goldstein, A.S. Effect of fiber diameter and orientation on fibroblast morphology and proliferation on electrospun poly(D,L-lactic-co-glycolic acid) meshes. Biomaterials 2006, 27, 5681-5688. [CrossRef] [PubMed]

21. Gentsch, R.; Pippig, F.; Schmidt, S.; Cernoch, P.; Polleux, J.; Boerner, H.G. Single-Step Electrospinning to Bioactive Polymer Nanofibers. Macromolecules 2011, 44, 453-461. [CrossRef]

22. Liao, S.; Murugan, R.; Chan, C.K.; Ramakrishna, S. Processing nanoengineered scaffolds through electrospinning and mineralization, suitable for biomimetic bone tissue engineering. J. Mech. Behav. Biomed. Mater. 2008, 1, 252-260. [CrossRef] [PubMed]

23. Li, W.; Tan, X.; Luo, T.; Shi, Y.; Yang, Y.; Liu, L. Preparation and characterization of electrospun PLA/PU bilayer nanofibrous membranes for controlled drug release applications. Integr. Ferroelectr. 2017, 179, 104-119. [CrossRef]

24. Lv, J.; Yin, X.; Zeng, Q.; Dong, W.; Liu, H.; Zhu, L. Preparation of carboxymethyl chitosan nanofibers through electrospinning the ball-milled nanopowders with poly (lactic acid) and the blood compatibility of the electrospun NCMC/PLA mats. J. Polym. Res. 2017, 24, 60. [CrossRef]

25. Zong, X.; Kim, K.; Fang, D.; Ran, S.; Hsiao, B.; Chu, B. Structure and process relationship of electrospun bioabsorbable nanofiber membranes. Polymer 2002, 43, 4403-4412. [CrossRef]

26. Kenawy, E.; Bowlin, G.; Mansfield, K.; Layman, J.; Simpson, D.; Sanders, E.; Wnek, G. Release of tetracycline hydrochloride from electrospun poly(ethylene-co-vinylacetate), poly(lactic acid), and a blend. J. Control. Release 2002, 81, 57-64. [CrossRef] 
27. Zeng, J.; Chen, X.; Xu, X.; Liang, Q.; Bian, X.; Yang, L.; Jing, X. Ultrafine fibers electrospun from biodegradable polymers. J. Appl. Polym. Sci. 2003, 89, 1085-1092. [CrossRef]

28. Zeng, J.; Aigner, A.; Czubayko, F.; Kissel, T.; Wendorff, J.; Greiner, A. Poly(vinyl alcohol) nanofibers by electrospinning as a protein delivery system and the retardation of enzyme release by additional polymer coatings. Biomacromolecules 2005, 6, 1484-1488. [CrossRef] [PubMed]

29. Kim, K.; Luu, Y.; Chang, C.; Fang, D.; Hsiao, B.; Chu, B.; Hadjiargyrou, M. Incorporation and controlled release of a hydrophilic antibiotic using poly(lactide-co-glycolide)-based electrospun nanofibrous scaffolds. J. Control. Release 2004, 98, 47-56. [CrossRef] [PubMed]

30. Xie, J.; Wang, C. Electrospun micro- and nanofibers for sustained delivery of paclitaxel to treat C6 glioma in vitro. Pharm. Res. 2006, 23, 1817-1826. [CrossRef] [PubMed]

31. Lyu, S.; Sparer, R.; Hobot, C.; Dang, K. Adjusting drug diffusivity using miscible polymer blends. J. Control. Release 2005, 102, 679-687. [CrossRef] [PubMed]

32. Zeng, J.; Yang, L.; Liang, Q.; Zhang, X.; Guan, H.; Xu, X.; Chen, X.; Jing, X. Influence of the drug compatibility with polymer solution on the release kinetics of electrospun fiber formulation. J. Control. Release 2005, 105, 43-51. [CrossRef] [PubMed]

33. Shi, Y.; Wei, Z.; Zhao, H.; Liu, T.; Dong, A.; Zhang, J. Electrospinning of Ibuprofen-Loaded Composite Nanofibers for Improving the Performances of Transdermal Patches. J. Nanosci. Nanotechnol. 2013, 13, 3855-3863. [CrossRef] [PubMed]

34. Zupancic, S.; Sinha-Ray, S.; Sinha-Ray, S.; Kristl, J.; Yarin, A.L. Long-Term Sustained Ciprofloxacin Release from PMMA and Hydrophilic Polymer Blended Nanofibers. Mol. Pharm. 2016, 13, 295-305. [CrossRef] [PubMed]

35. Zupancic, S.; Sinha-Ray, S.; Sinha-Ray, S.; Kristl, J.; Yarin, A.L. Controlled Release of Ciprofloxacin from Core-Shell Nanofibers with Monolithic or Blended Core. Mol. Pharm. 2016, 13, 1393-1404. [CrossRef] [PubMed]

36. Khansari, S.; Duzyer, S.; Sinha-Ray, S.; Hockenberger, A.S.; Yarin, A.L.; Pourdeyhimi, B. Two-Stage Desorption-Controlled Release of Fluorescent Dye and Vitamin from Solution-Blown and Electrospun Nanofiber Mats Containing Porogens. Mol. Pharm. 2013, 10, 4509-4526. [CrossRef] [PubMed]

37. Wolfe, P.S.; Lochee, Y.; Jhurry, D.; Bhaw-Luximon, A.; Bowlin, G.L. Characterization of Electrospun Novel Poly(ester-ether) Copolymers: 1,4-Dioxan-2-one and D,L-3-Methyl-1,4-dioxan-2-one. J. Eng. Fibers Fabr. 2011, 6, 60-69.

38. Meng, Z.X.; Zheng, W.; Li, L.; Zheng, Y.F. Fabrication, characterization and in vitro drug release behavior of electrospun PLGA/chitosan nanofibrous scaffold. Mater. Chem. Phys. 2011, 125, 606-611. [CrossRef]

39. Zhang, Y.; Wang, X.; Feng, Y.; Li, J.; Lim, C.; Ramakrishna, S. Coaxial electrospinning of (fluorescein isothiocyanate-conjugated bovine serum albumin)-encapsulated poly(epsilon-caprolactone) nanofibers for sustained release. Biomacromolecules 2006, 7, 1049-1057. [CrossRef] [PubMed]

40. Wang, C.; Yan, K.; Lin, Y.; Hsieh, P.C.H. Biodegradable Core/Shell Fibers by Coaxial Electrospinning: Processing, Fiber Characterization, and Its Application in Sustained Drug Release. Macromolecules 2010, 43, 6389-6397. [CrossRef]

41. Wu, J.; Wang, N.; Zhao, Y.; Jiang, L. Electrospinning of multilevel structured functional micro-/nanofibers and their applications. J. Mater. Chem. A 2013, 1, 7290-7305. [CrossRef]

42. Varabhas, J.S.; Chase, G.G.; Reneker, D.H. Electrospun nanofibers from a porous hollow tube. Polymer 2008, 49, 4226-4229. [CrossRef]

43. Tran, C.; Kalra, V. Co-continuous nanoscale assembly of Nafion-polyacrylonitrile blends within nanofibers: A facile route to fabrication of porous nanofibers. Soft Matter 2013, 9, 846-852. [CrossRef]

44. Cao, S.; Hu, B.; Liu, H. Synthesis of pH-responsive crosslinked poly[styrene-co-(maleic sodium anhydride)] and cellulose composite hydrogel nanofibers by electrospinning. Polym. Int. 2009, 58, 545-551. [CrossRef]

45. Qi, M.; Li, X.; Yang, Y.; Zhou, S. Electrospun fibers of acid-labile biodegradable polymers containing ortho ester groups for controlled release of paracetamol. Eur. J. Pharm. Biopharm. 2008, 70, 445-452. [CrossRef] [PubMed]

46. Kim, Y.; Ebara, M.; Aoyagi, T. Temperature-responsive electrospun nanofibers for 'on-off' switchable release of dextran. Sci. Technol. Adv. Mater. 2012, 13, 064203. [CrossRef] [PubMed]

47. Fu, G.; Xu, L.; Yao, F.; Li, G.; Kang, E. Smart Nanofibers with a Photoresponsive Surface for Controlled Release. ACS Appl. Mater. Interfaces 2009, 1, 2424-2427. [CrossRef] [PubMed] 
48. Abidian, M.; Kim, D.; Martin, D. Conducting-polymer nanotubes for controlled drug release. Adv. Mater. 2006, 18, 405-409. [CrossRef] [PubMed]

49. Tan, S.; Wendorff, J.; Pietzonka, C.; Jia, Z.; Wang, G. Biocompatible and biodegradable polymer nanofibers displaying superparamagnetic properties. ChemPhysChem 2005, 6, 1461-1465. [CrossRef] [PubMed]

50. Wang, A.; Singh, H.; Hatton, T.; Rutledge, G. Field-responsive superparamagnetic composite nanofibers by electrospinning. Polymer 2004, 45, 5505-5514. [CrossRef]

51. Chen, H.; Hsieh, Y. Ultrafine hydrogel fibers with dual temperature- and $\mathrm{pH}$-responsive swelling behaviors. J. Polym. Sci. Part A Polym. Chem. 2004, 42, 6331-6339. [CrossRef]

52. Li, L.; Hsieh, Y. Ultra-fine polyelectrolyte hydrogel fibers from poly(acrylic acid)/poly(vinyl alcohol). Nanotechnology 2005, 16, 2852-2860. [CrossRef]

53. Sanson, C.; Schatz, C.; Le Meins, J.; Soum, A.; Thevenot, J.; Garanger, E.; Lecommandoux, S. A simple method to achieve high doxorubicin loading in biodegradable polymersomes. J. Control. Release 2010, 147, 428-435. [CrossRef] [PubMed]

54. Sato, S.; Gondo, D.; Wada, T.; Kanehashi, S.; Nagai, K. Effects of various liquid organic solvents on solvent-induced crystallization of amorphous poly(lactic acid) film. J. Appl. Polym. Sci. 2013, 129, 1607-1617. [CrossRef]

55. Li, L.; Jiang, Z.; Xu, J.; Fang, T. Predicting Poly(vinyl pyrrolidone)'s Solubility Parameter and Systematic Investigation of the Parameters of Electrospinning with Response Surface Methodology. J. Appl. Polym. Sci. 2014, 131, 40304. [CrossRef]

56. Hansen, C.M. Hansen Solubility Parameters: A User's Handbook, 2nd ed.; CRC Press: Boca Raton, FL, USA, 2007.

57. Wenzel, R.N. Resistance of Solid Surfaces to Wetting by Water. Ind. Eng. Chem. 1936, 28, 988-994. [CrossRef]

(C) 2018 by the authors. Licensee MDPI, Basel, Switzerland. This article is an open access article distributed under the terms and conditions of the Creative Commons Attribution (CC BY) license (http:/ / creativecommons.org/licenses/by/4.0/). 